\title{
Effects of Speed on Coupled Sweep and Camber in Morphing Wings
}

\author{
Lawren L. Gamble ${ }^{1}$, Amin Moosavian ${ }^{2}$, and Daniel J. Inman ${ }^{3}$ \\ Department of Aerospace Engineering, University of Michigan, Ann Arbor, MI, 48109
}

\begin{abstract}
Like birds, bats, and insects, a benefit of morphing aircraft is their ability to adapt to a variety of flight conditions by changing the geometry of their wings, unlike their traditional counterparts which remain designed and optimized typically for a single flight condition. Here we study the coupled effect of multi-scale morphing, namely sweep and camber, for varying velocities. Nine different wing configurations are considered consisting of combinations of three sweep angles and three airfoil profiles. The three sweep configurations include minimally, moderately, and highly swept planforms. The airfoil profiles considered include a NACA 0012 airfoil, a conventionally cambered airfoil, and a reflex cambered airfoil. The study is based on numerical simulations conducted using a Reynolds-averaged Navier-Stokes (RANS) turbulence model for low-Reynolds-number flow. The results for the simulations are presented and discussed with a particular focus on gliding behavior during steady level flight. From the numerical results, there is a clear indication that there are considerable benefits in the proposed multi-scale morphing, modifying the wing and the airfoil shapes at varying velocities.
\end{abstract}

\section{Nomenclature}

$\begin{array}{ll}A R & =\text { wing aspect ratio } \\ b & =\text { wing span } \\ c & =\text { airfoil chord length } \\ C_{D} & =\text { nondimensional drag coefficient } \\ C_{L} & =\text { nondimensional lift coefficient } \\ D & =\text { drag force } \\ L & =\text { lift force } \\ v & =\text { free stream velocity } \\ S & =\text { wing planform area } \\ \rho & =\text { fluid density }\end{array}$

\section{Introduction}

B IOLOGY has long inspired engineering design and this is particularly true within the field of aerospace engineering. This is in part due to the fact that birds are masters of the skies in ways which Unmanned Aerial Vehicle (UAV) designers can only dream, exhibiting supreme efficiency and precise control as a result of both large scale and small scale geometric tailoring. This predominantly includes wing sweep and camber, in addition to tail spread, twist, and pitch ${ }^{1,2}$. Sweep is perhaps the most dramatic change in wing geometry and has been shown to extend the flight envelope by providing a solution to efficient gliding flight with extended wings, in addition to diving flight and increased turning rate with swept wings ${ }^{3}$. This is partly due to the effects of reduced planform area with increasing wing sweep. Secondly, wing camber can be adapted by changing wing posture ${ }^{4}$ which affects the wing's aerodynamic properties namely increasing lift and drag with increasing camber ${ }^{5}$. In other words, birds morph their bodies to adapt to their environment giving them an advantage to traditional aircraft, particularly when it comes to operating at multiple flight speeds.

\footnotetext{
${ }^{1}$ Ph.D. Candidate, Aerospace Engineering, 1320 Beal Ave., AIAA Member

${ }^{2}$ Post-Doctoral Fellow, Aerospace Engineering, 1320 Beal Ave., AIAA Member

${ }^{3}$ Collegiate Professor and Chair, Aerospace Engineering, 1320 Beal Ave., AIAA Fellow
}

American Institute of Aeronautics and Astronautics 
From these investigations on bird wing sweep and camber, it is evident that morphology provides distinct advantages over a range of flight speeds. These trends have also been investigated in aircraft. At low speeds, relative to both aircraft and small UAVs, increasing wing sweep provides increases in longitudinal stability while low swept wings provide higher lift-drag ratios, as the lift-curve slope decreases with increasing sweep ${ }^{6,7}$. At the UAV scale, Hall et al. also found that, for a variable swept wing concept which experienced minimal changes in planform area, increasing sweep tends to reduce the drag coefficient due to the reduction in frontal area which reduces the parasitic drag component. However, the effect of wind speed was minimal compared to the sweep effects and only demonstrated a slight decrease in drag with the exception of a level flight assumption which demonstrated that highly swept wings experienced a significant reduction in drag with increasing wind speed. Furthermore, the airfoil geometry is well known to impact the aerodynamic characteristics. Increasing the camber of the wing increases both lift and drag, while increasing wind speed increases the maximum lift while keeping the lift-curve slope the same. Lastly, a reflexed airfoil shape has been shown to provide better lift-to-drag ratios at large angles of attack near stall ${ }^{8}$.

With the synchronized progression of innovative UAV designs, avian studies, and smart materials, new morphing concepts utilizing the benefits of these geometries in an adaptive manner have been realized. A large portion of these morphing wing concepts have focused on airfoil shape which, as mentioned prior, heavily impacts aerodynamic characteristics. One of the most influential camber morphing concepts within the smart materials community is the cascading bimorph developed by Bilgen et al. ${ }^{9}$. This design implemented the curved actuation performance of Macro Fiber Composites (MFCs), a piezoelectric actuator, to achieve a smooth trailing edge camber deformation with rapid actuation time. Pankonien et al. later extended this design to the Synergistic Smart Morphing Aileron (SSMA), which couples an MFC trailing edge actuator with an antagonistic hinge using Shape Memory Alloy (SMA) wires ahead of the MFCs ${ }^{10}$. When both the MFC and SMA are actuated in the same direction, this allows for a greater range of tip deflection, with the small range of deflection being a shortcoming of many morphing wing designs, compounding the effects of the SMA deflection and the MFC deflection. Conversely, when they are actuated in opposing directions, the airfoil experiences reflex camber.

In this work, we aim to investigate the coupled aerodynamic effect of wind speed and multi-scale morphing, defined as exhibiting both large scale changes in geometry such as wing sweep in addition to small scale deformations such as two-dimensional changes in airfoil shape. This was achieved by conducting simulations using a Reynoldsaveraged Navier-Stokes (RANS) turbulence model for a variety of wing configurations with combinations of wing sweep and camber, as will be discussed in the following section. These configurations were tested at multiple wind speeds in order to investigate the effects on multi-scale morphing.

\section{Multi-scale Morphing Concept}

The multi-scale morphing concept harnesses the biological advantages found in many natural flyers in the hopes of achieving more efficient flight over a range of operating conditions. Here, we focus on the effects of wing sweep and camber which are known to have a significant impact on aerodynamic characteristics. The geometry for the wing configurations were chosen to resemble the work of Lentink et al. and Pankonien et al. for consistency and comparability. The following discussion will begin with a detailed discussion of the small scale morphing geometry, followed by the large scale morphing geometry.

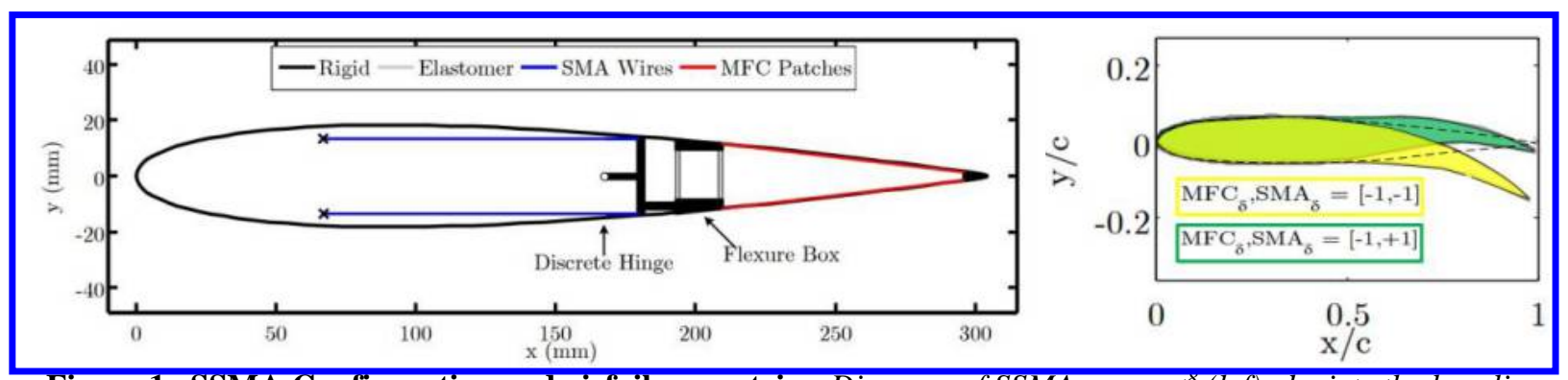

Figure 1. SSMA Configuration and airfoil geometries. Diagram of SSMA concept ${ }^{8}$ (lef), depicts the baseline, monotonic and reflex configurations tested. (right)

American Institute of Aeronautics and Astronautics 
The airfoil geometries were chosen to represent the exact geometries of the SSMA design developed by Pankonien et al. Three geometries were used, representing the extremes of this concept's actuation range. The primary or baseline geometry was a NACA0012 symmetric airfoil. This represents an undeformed airfoil shape and facilitates comparison across airfoil geometries as well as sweep angles. The second geometry represents monotonic actuation, described by the MFC and SMA mechanisms actuating at their maximum potential in the same direction. This produces a cambered airfoil where the tip deflection is compounded due to the effects of both morphing mechanisms. In terms of a normalized signal, this would be represented by $[-1,-1]$, representing tip deflection in the -y direction which inherently produces positive lift. Lastly, the third geometry represents reflexed actuation, described by the MFC and SMA mechanisms actuating at their maximum potential in opposite directions. This produces a reflexed airfoil where the camber line is serpentine. The normalized signal is represented by $[1,-1]$. This configuration was chosen, as opposed to $[-1,1]$, since the camber of the MFC has a stronger influence on the aerodynamic properties, and a positive lift with positive angle of attack was desired for comparison purposes. Figure 1 shows the monotonic and the reflexed airfoil shapes.

The sweep geometries were chosen to represent the swept wing shapes used by Lentink et al. which were derived from the actual wing shape of swifts. However, here we aim to investigate the sole effects of sweep as opposed to the coupled effects of sweep and planform area change. Accordingly, the minimally swept wing at $5^{\circ}$ represents the exact geometry used by Lentink et al; however, the higher sweep angles $\left(30^{\circ}\right.$ and $\left.55^{\circ}\right)$ do not align with those reported by Lentink et al. The higher sweep configurations reported by Lentink et al. combine changes to both the sweep angle as well as the planform area. Although in nature the changes in sweep and wing area are typically coupled, this makes it difficult to attribute specific trends to either solely to the change in the sweep or the area. In order to isolate the sole effects of sweep in the geometries studied here, all the planform areas and root chords are identical.

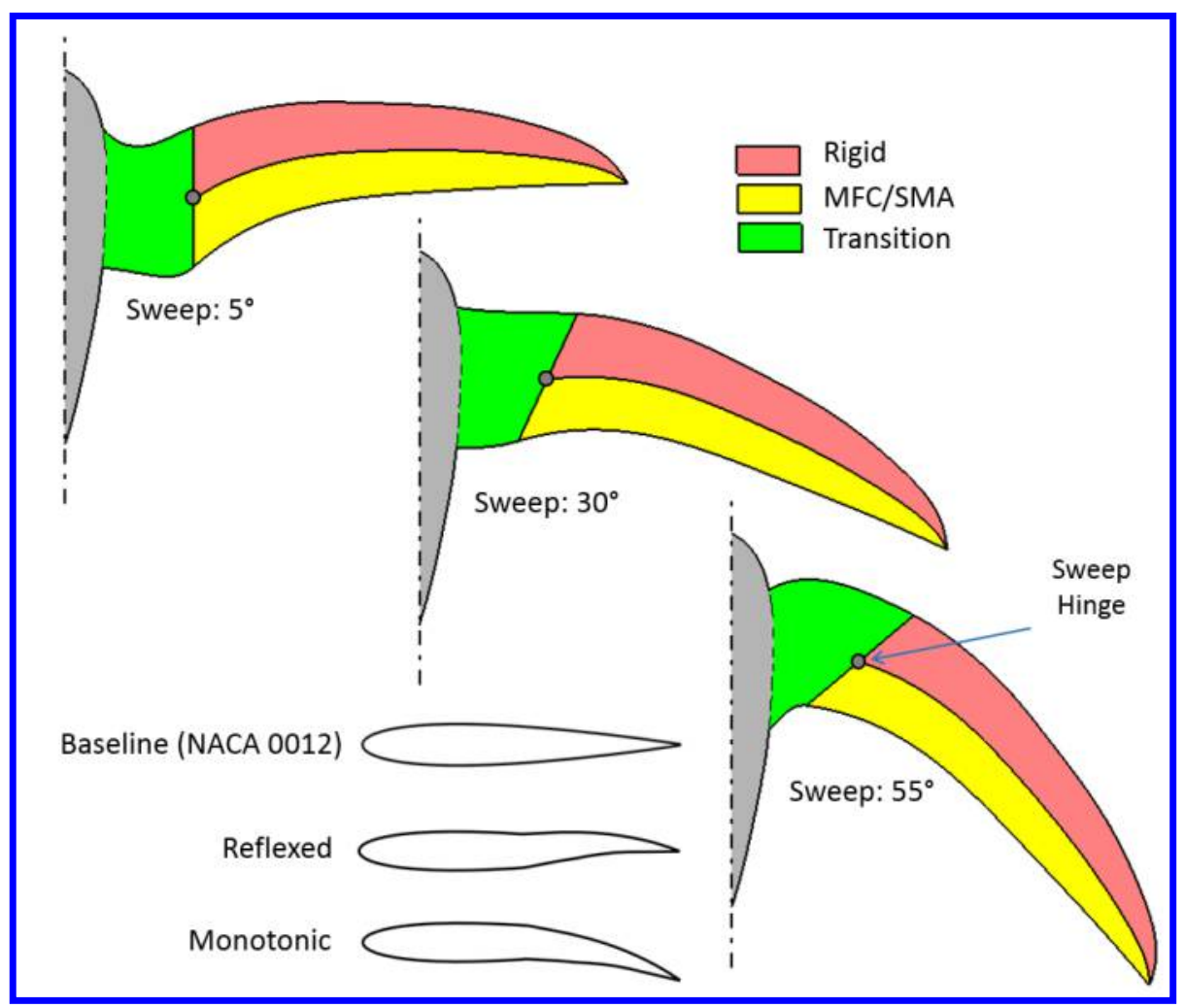

Figure 2. Morphing Mechanism. Proposed morphing configuration incorporating a sweep morphing mechanism and the SSMA camber morphing concept

The wing root chord used for this study is $0.053 \mathrm{~m}$, and the planform area is $7.07 \times 10^{-3} \mathrm{~m}^{2}$. As shown in Fig. 2, the planform is broken up into three regions. The first is the rigid section, which will not see any change in its profile,

American Institute of Aeronautics and Astronautics 
but will undergo changes in sweep along with the rest of the wing. The second region occupies the zone between the mid chord all the way to the trailing edge of the wing. This region will undergo changes both in the profile and the sweep. The third region (transition), is utilized to generate a smooth geometric transition between the morphing portion of the wing and the fuselage. The shape of the transition region was driven by respecting the following requirements: maintaining a constant chord length at the wing root; maintaining tangent-continuity where the leading and trailing edges of the rest of the wing meet the transition region; and maintaining a constant area among all three planform configurations. Figure 3 illustrates the three planforms, showing the change in sweep, and the three airfoil profiles, used in the study. The three sweep angles used are $5^{\circ}, 30^{\circ}$, and $55^{\circ}$, while the airfoil profiles are baseline (NACA0012), monotonic, and reflexed. The combination of the three planforms and the three profiles leads to a total of nine configurations.

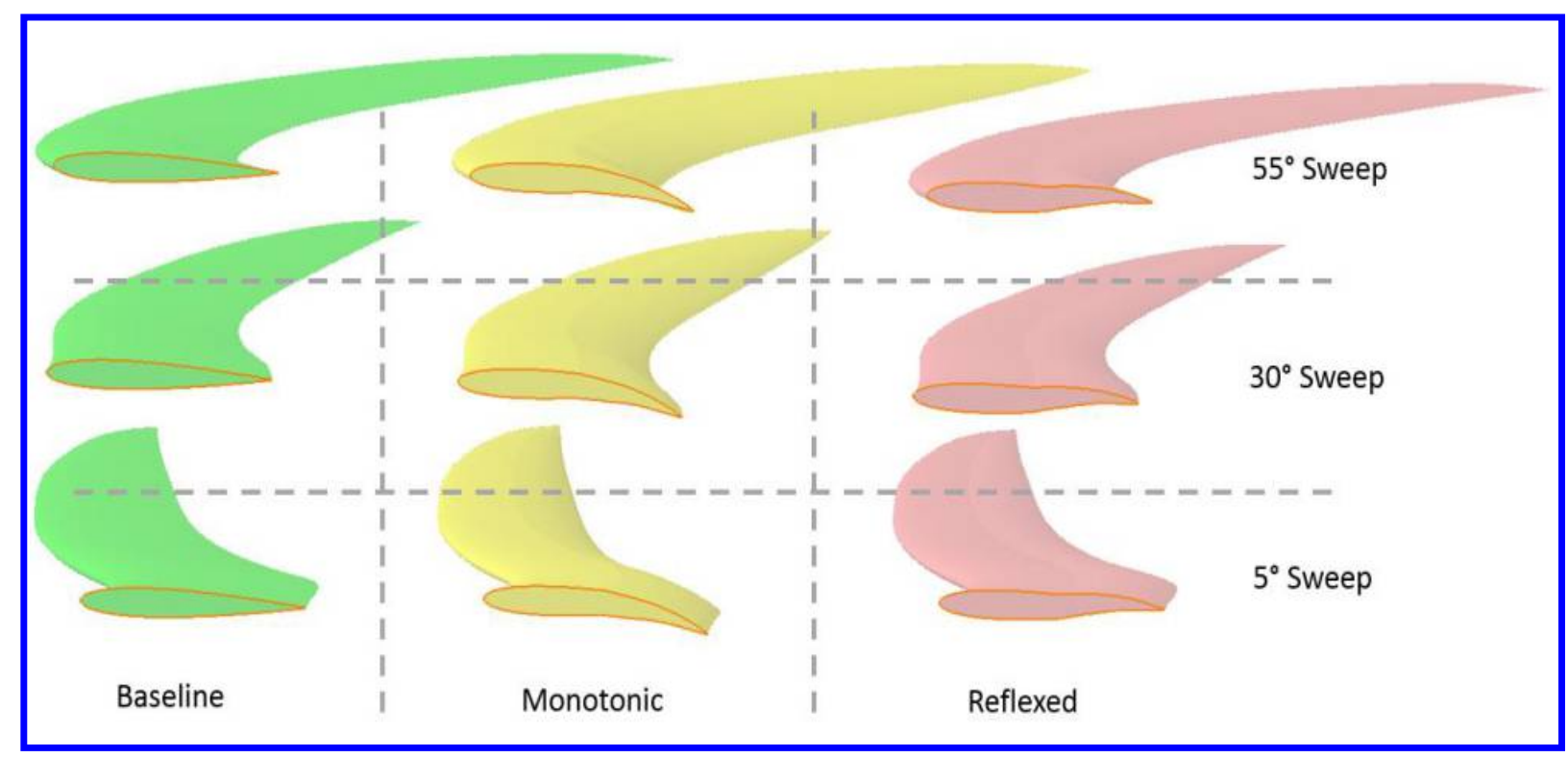

Figure 3. Wing Configurations. All 9 wing configurations as the result of combinations of 3 airfoil geometries and 3 sweep angles.

\section{RANS Simulations}

Numerical flow simulations were conducted using a Reynolds-averaged Navier-Stokes (RANS) turbulence model in order to analyze the low-Reynolds number aerodynamic performance of all 9 wing shapes at wind speeds of 10, 15, and $20 \mathrm{~m} / \mathrm{s}$ which are representative of a small UAV or bird. Due to the multi-scale morphing properties of these configurations, a three-dimensional time-steady simulation was required. Although the effects of camber and sweep deformation would be transient in nature, this work is concerned solely with the steady-state aerodynamic properties. The simulation simultaneously solves the pressure and momentum equations, utilizing a coupled pressure-based solver. Although multiple wind speeds are considered in this analysis, they all represent fairly low Reynolds numbers. Thus, a standard $\mathrm{k}-\omega$ turbulence model was chosen.

The wing was discretized into a three-dimensional mesh of tetrahedral finite volume elements. Due to the complex geometries of these configurations, an unstructured mesh was chosen for simplicity. The mesh was locally refined nearest the regions of high curvature. This translates to a high density mesh at the wing's trailing edge and tip, with medium density at the leading edge. A demonstration of the mesh can be seen in Fig. 4. Furthermore, for simplification of the calculations a symmetry plane was introduced at the wing root which ensures the flow does not envelop the root without requiring the presence of a wall. 


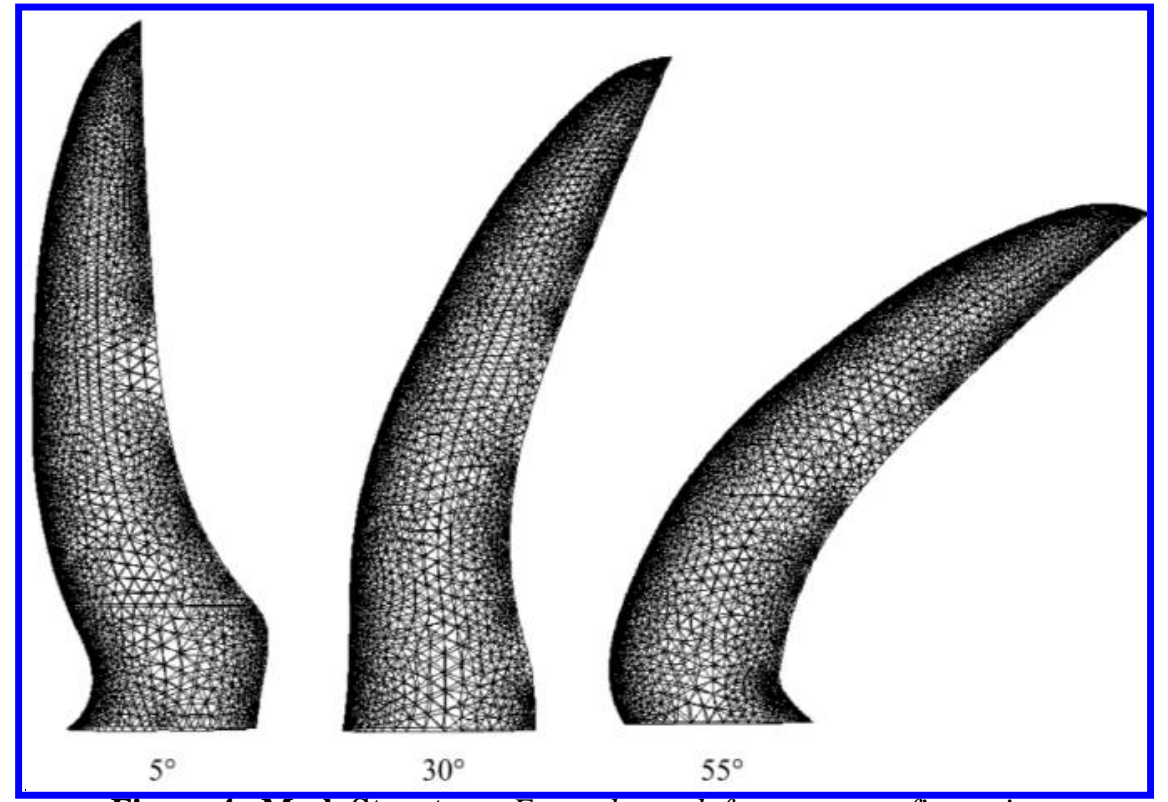

Figure 4. Mesh Structure. Example mesh for swept configurations

Aerodynamic characteristics were determined for each configuration at $10 \mathrm{~m} / \mathrm{s}, 15 \mathrm{~m} / \mathrm{s}$, and $20 \mathrm{~m} / \mathrm{s}$ across angles of attack ranging from $-25^{\circ}$ to $25^{\circ}$. Two quantities were of primary interest: the lift and drag coefficients. These nondimensional parameters are written as follows:

$$
\begin{aligned}
& C_{L}=\frac{L}{\frac{1}{2} \rho v^{2} S} \\
& C_{D}=\frac{D}{\frac{1}{2} \rho v^{2} S}
\end{aligned}
$$

Since these parameters are normalized by the fluid and geometric properties, this allows for comparison across configurations.

\section{Results and Discussion}

\section{A. Aerodynamic Characterization}

Figure 5 shows the raw data from the simulations in terms of the lift and drag coefficients, grouped by wind speed. From this a few trends are evident. The monotonic airfoil has a high lift performance across all angles of attack regardless of wind speed and sweep, in addition to a higher drag coefficient. However, the highly swept wing exhibits a substantial loss in lift when compared to the other two sweep angles. This is in part due to a reduction in the liftcurve slope which is a trend seen in low aspect ratio wings. Careful observation reveals that this trend is also present in the other two airfoil configurations. Hence, the wing sweep effectively reduces the wing aspect ratio. This can be further validated using the general equation for aspect ratio which is given as

$$
A R=\frac{b^{2}}{s}
$$

For a wing of fixed length, the wing span decreases with increasing sweep. Thus from Eq. 3 it is clear that the aspect ratio effectively decreases with increasing sweep. Furthermore, though not as prominent as the effects of airfoil shape, the drag coefficient decreases with increasing wing sweep for positive angles of attack and the lift increases with wind speed across all configurations. These trends were anticipated, as was mentioned in the introduction. However, further analysis is required to fully detail when each of these configurations may be useful. This is particularly evident given the minor differences in drag across wind speeds and the crossover in the lift between the cases at large angles of attack.

American Institute of Aeronautics and Astronautics 


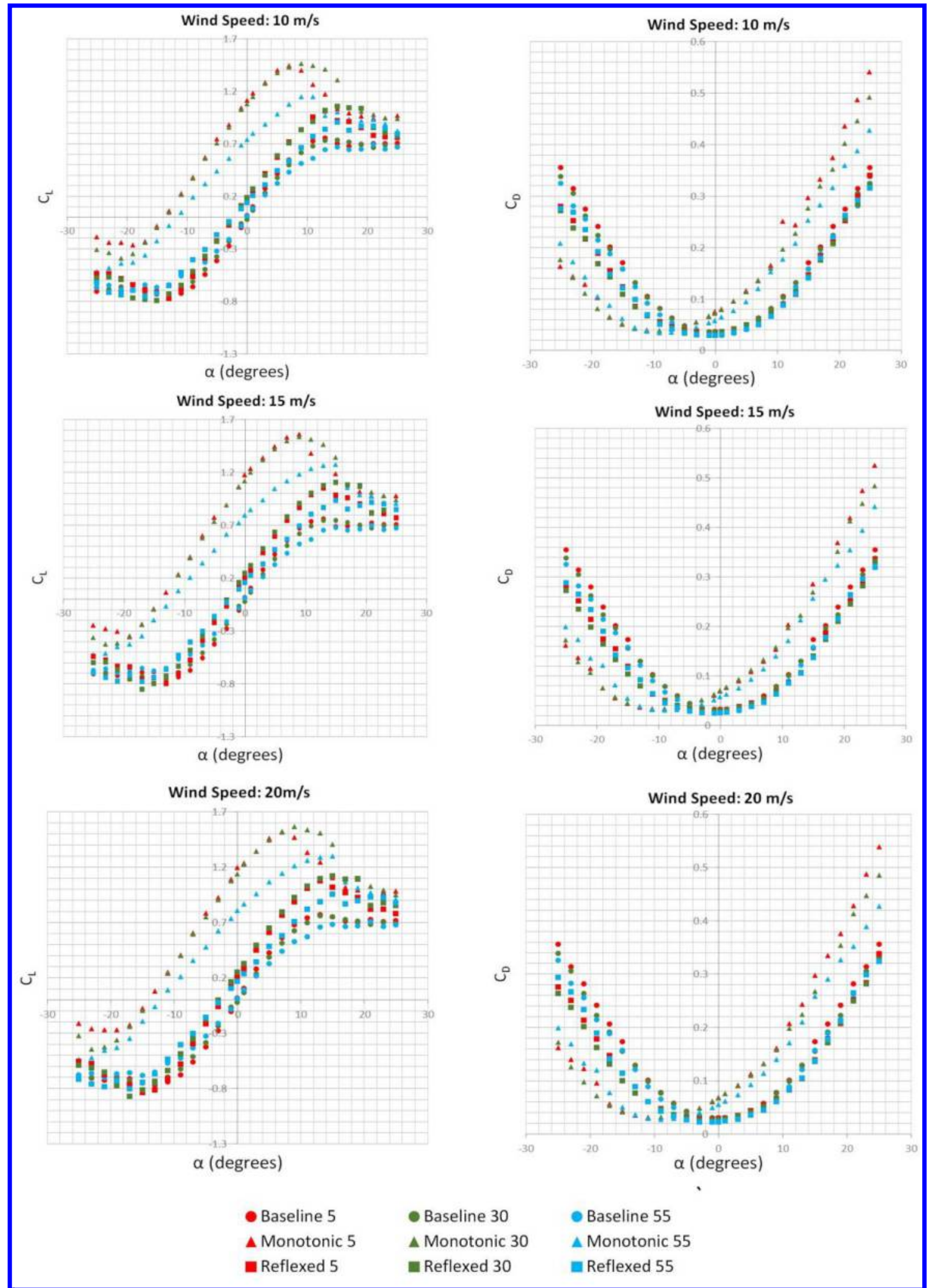

Figure 5. Lift and Drag Coefficients. Comparison of lift and drag coefficients across configurations, grouped by wind speed 
Figure 6 illustrates the glide ratio as a function of the angle of attack for all nine configurations grouped together based on velocity. As expected, the glide ratio increases with wind speed across all configurations. However, this trend is particularly prominent in the reflexed wings. The reflexed wings also outperform both the monotonic and baseline wings at high angles of attack near and post-stall. This can be attributed to the airfoil's ability to generate lift with relatively low drag consequences. This region of interest widens substantially as the wind speed increases, as is shown by the outlined areas of the curve. Lastly, while most configurations demonstrate that swept wings tend to reduce the glide ratio, the reflexed wings experience a crossover phenomenon which indicates that the coupling effect of sweep and reflex camber are not directly dependent upon the wind speed. This region is marked on each plot by an asterisk. It shows that at $10 \mathrm{~m} / \mathrm{s}$, the $30^{\circ}$ and $55^{\circ}$ sweep reflexed wings perform fairly equally. But as the wind speed increases to both $15 \mathrm{~m} / \mathrm{s}$ and $20 \mathrm{~m} / \mathrm{s}$, the $30^{\circ}$ sweep wing shows a substantial increase in glide ratio compared to the $55^{\circ}$ wing. While it was shown that the highly swept wing does exhibit a smaller drag coefficient, its substantial reduction in lift coefficient proves to be its downfall with respect to the glide ratio. However, the dependence of these aerodynamic parameters on the angle of attack is not always useful.

Another way of viewing this data is through the drag polar which eliminates any dependence upon the angle of attack. These plots are fairly consistent across wind speeds and any trends present have already been noted in the prior analysis. Thus, only the $20 \mathrm{~m} / \mathrm{s}$ case is shown below in Fig. 6d. This very clearly shows that the ability of the wing to morph its airfoil shape between baseline, monotonic and reflex substantially widens the performance envelope. However given all of this information, a question which remains unanswered is when each of these configurations is useful.

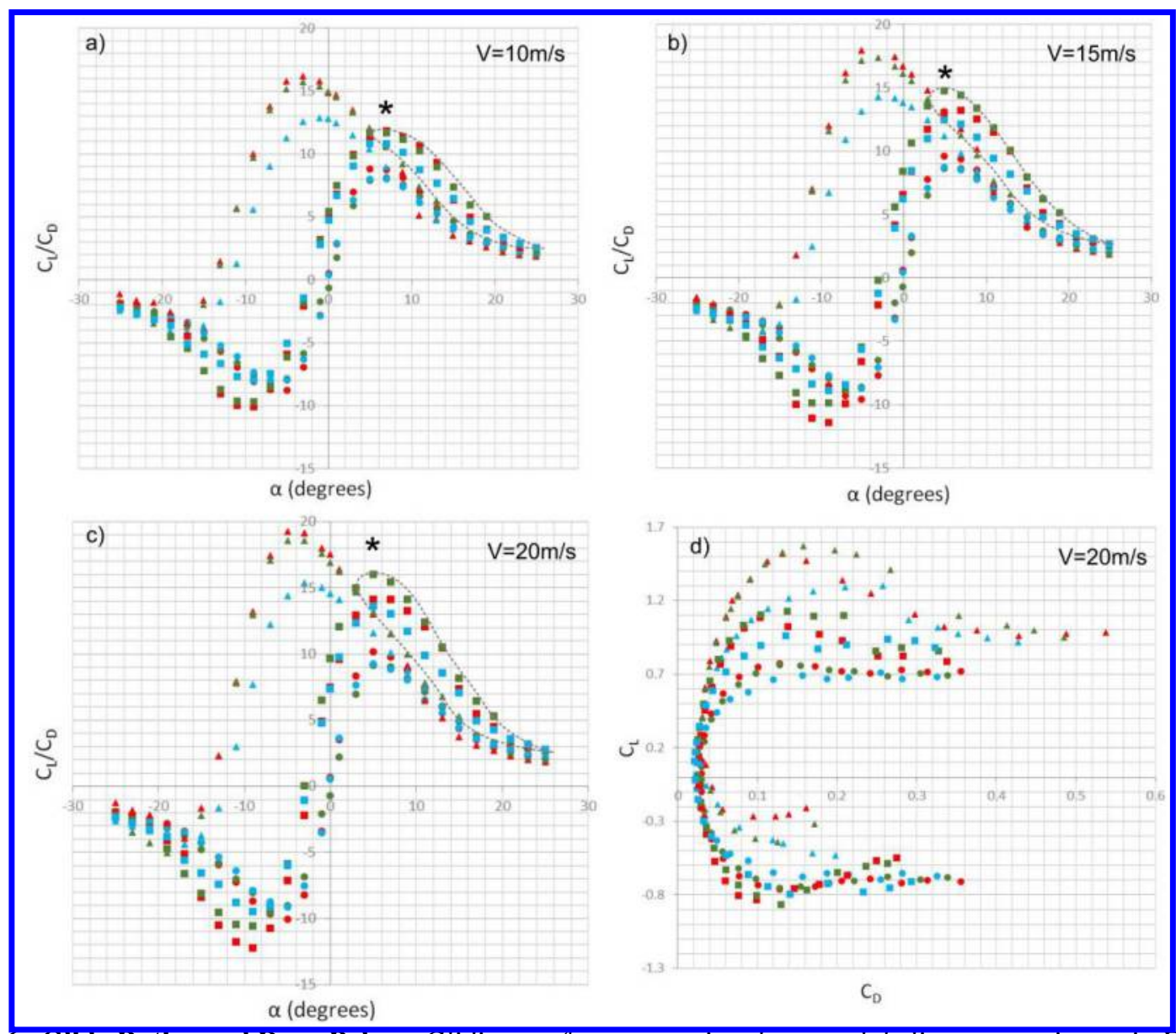

Figure 6. Glide Ratios and Drag Polars. Gliding performance using the same labeling convention as in Fig. 5 for a) $10 \mathrm{~m} / \mathrm{s}$ b) $15 \mathrm{~m} / \mathrm{s} \mathrm{c)} 20 \mathrm{~m} / \mathrm{s}$; and d) Drag polar at $20 \mathrm{~m} / \mathrm{s}$ 


\section{B. Steady Level Flight}

One approach to compare the effectiveness of the various configurations in relation to velocity is to inspect the glide ratio $\left(C_{L} / C_{D}\right)$ required to maintain Steady Level Flight (SLF) for a given velocity. In SLF, with the total lift being equal to the weight of the flying body, one can use a given weight to calculate the lift coefficient required to maintain SLF using Eq. 1. Here a weight (W) of $1 \mathrm{~N}$ is assumed. Using $\mathrm{S}=7.07 \times 10^{-3} \mathrm{~m}^{2}$ and $\rho=1.225 \mathrm{~kg} / \mathrm{m}^{3}$, the lift for one wing can be calculated for the three velocities simulated for this study. By finding the corresponding drag (with the aid of the drag polars generated from the simulations) for each velocity and wing/airfoil configuration, the glide ratio can be obtained as a function of the velocity. Using the glide ratio as a measure of optimality, the most optimal configuration can be identified for each velocity. Figure 8 illustrates the highest glide ratio that could be obtained for $\mathrm{W}=1 \mathrm{~N}$ and the corresponding sweep and camber. The weight here refers to the total weight of the body, balanced by lift produced by two symmetric wings.

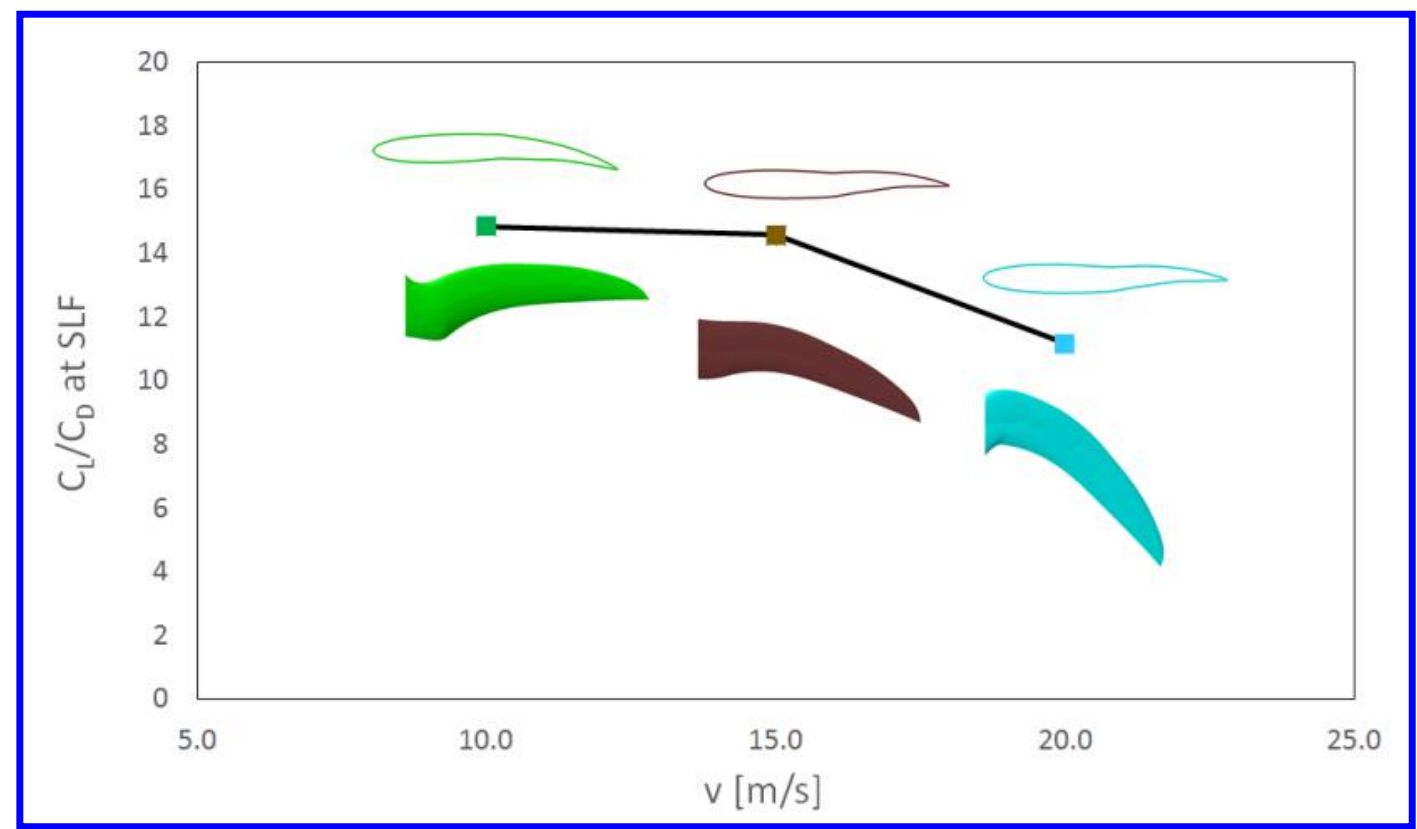

Figure 7. Steady Flight with respect to Speed. Most optimal configurations for $W=1 \mathrm{~N}$ at $10 \mathrm{~m} / \mathrm{s}, 15 \mathrm{~m} / \mathrm{s}$, and 20

$$
\mathrm{m} / \mathrm{s}
$$

As it can be seen from Fig. 7 there is a clear advantage in multi-scale morphing, allowing for changes in the planform as well as the airfoil shape. As the velocity increases, the sweep needs to be increased to maintain the highest possible glide ratio. For $\mathrm{W}=1 \mathrm{~N}$, this corresponds to a $5^{\circ}$ sweep at $10 \mathrm{~m} / \mathrm{s}$, a $30^{\circ}$ sweep at $15 \mathrm{~m} / \mathrm{s}$, and a $55^{\circ}$ sweep at $20 \mathrm{~m} / \mathrm{s}$. These variations in sweep are accompanied by changes in the airfoil profile, with $10 \mathrm{~m} / \mathrm{s}$ favoring the monotonic shape, and the $15 \mathrm{~m} / \mathrm{s}$ and $20 \mathrm{~m} / \mathrm{s}$ favoring the reflexed shape. Furthermore, Figs. 8 through 10 illustrate the sensitivity of the optimal wing/airfoil configuration to the weight in SLF at $10 \mathrm{~m} / \mathrm{s}, 15 \mathrm{~m} / \mathrm{s}$, and $20 \mathrm{~m} / \mathrm{s}$, respectively.

The airfoil shape of a bird wing is hypothesized to change passively due to aerodynamic loads which deform the feather structures from a conventionally cambered shape into a reflex cambered shape. This hypothesis can be reaffirmed by the trend observed in the study presented here. With the increase in velocity, the change from monotonic to reflex brings about the highest possible glide ratio, as shown in Fig. 7. Furthermore, this change in the airfoil is accompanied by an increase in the sweep angle. 


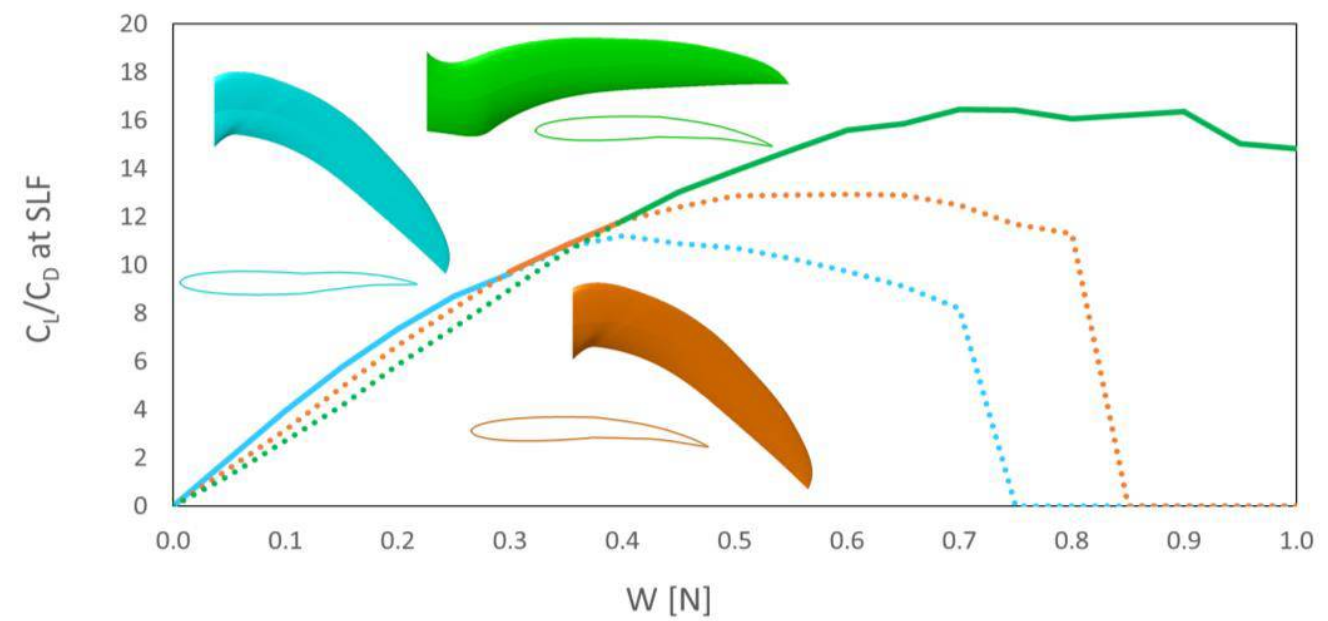

Figure 8. Steady Level Flight 10m/s. Most optimal configurations for $S L F$ at $10 \mathrm{~m} / \mathrm{s}$

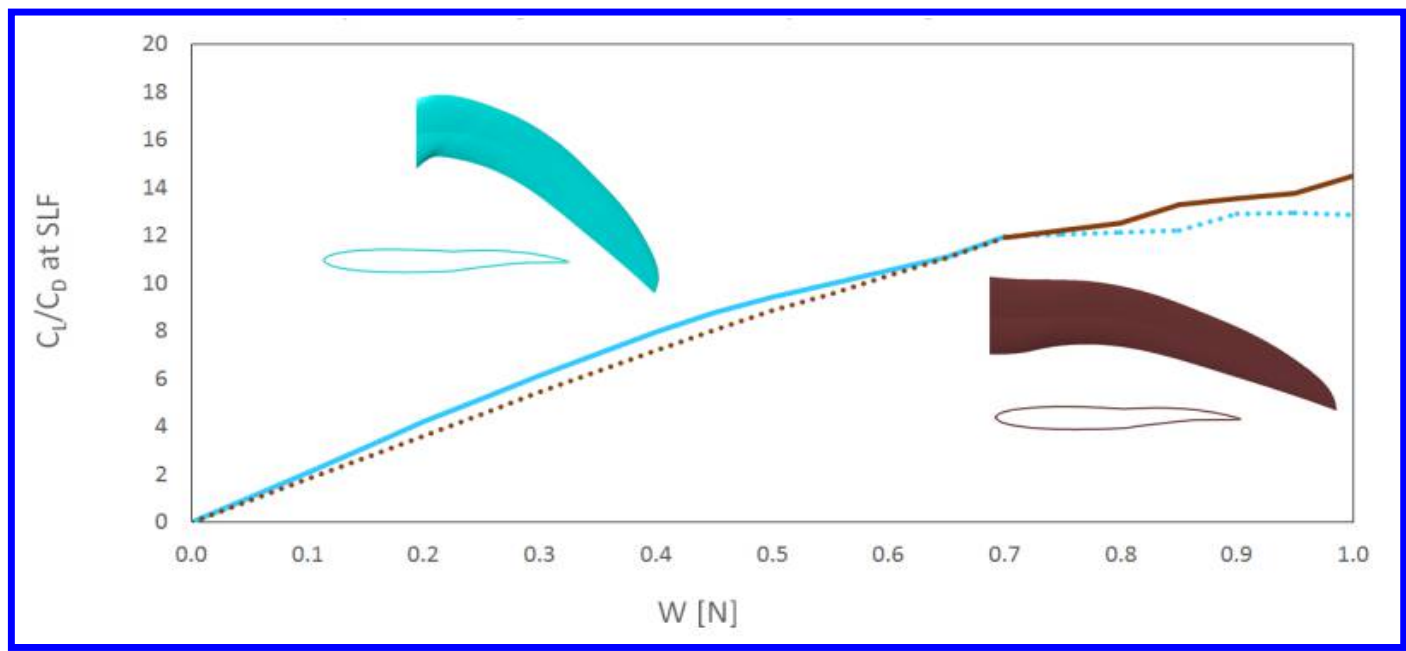

Figure 9. Steady Level Flight $15 \mathrm{~m} / \mathrm{s}$. Most optimal configurations for $S L F$ at $15 \mathrm{~m} / \mathrm{s}$

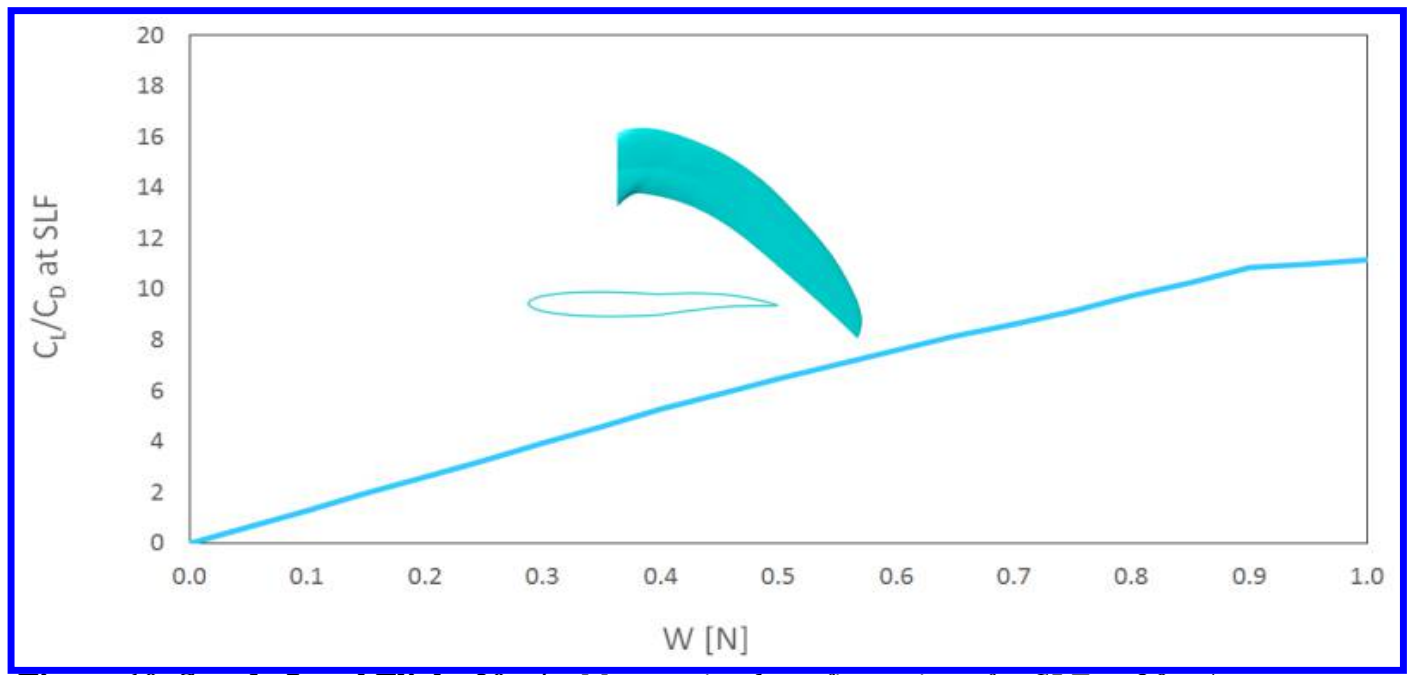

Figure 10 . Steady Level Flight $20 \mathrm{~m} / \mathbf{s}$. Most optimal configurations for SLF at $20 \mathrm{~m} / \mathrm{s}$

American Institute of Aeronautics and Astronautics 


\section{Conclusion}

Based on a bio-inspired design a study was presented to evaluate the impact of morphing coupled sweep and camber at three different velocities. The planform geometry was based on the wing of a swift, while the airfoil geometry was based on the SSMA morphing airfoil concept. A total of nine configurations were studied at velocities of $10 \mathrm{~m} / \mathrm{s}, 15 \mathrm{~m} / \mathrm{s}$, and $20 \mathrm{~m} / \mathrm{s}$. The numerical results suggest that there is a clear advantage in morphing both the sweep and the camber at varying velocities. As future work, it is planned to expand the velocity beyond the $10-20 \mathrm{~m} / \mathrm{s}$ range. Additionally, some experimental work is planned to confirm the trends presented in this paper.

\section{Acknowledgments}

This work was supported in part by the US Air Force Office of Scientific Research (AFOSR) under grant number FA9550-161-0087, titled “Avian-Inspired Multifunctional Morphing Vehicles" monitored by Dr. B.L. Lee, and in part by the Natural Sciences and Engineering Research Council of Canada (NSERC).

\section{References}

${ }^{1}$ Carruthers, A.C., Thomas, A.L., and Taylor, G.K., "Automatic Aeroelastic Devices in the Wings of a Steppe Eagle Aquila Nipalensis," Journal of Experimental Biology, Vol. 201. No. 23, 2007, pp. 4136-4149.

${ }^{2}$ Gillies, J.A., Thomas, A.L., and Taylor, G.K., "Soaring and Manoeuvring Flight of a Steppe Eagle Aquila Nipalensis," Journal of Avian Biology, Vol. 42, No. 5, 2011, pp. 377-386.

${ }^{3}$ Lentink, D., et al., "How Swifts Control Their Glide Performance with Morphing Wings," Nature, Vol. 446, 2007, pp. 10821085 .

${ }^{4}$ Bachmann, T., et al., "Barn Owl Flight," Nature-Inspired Fluid Mechanics, Springer Berlin Heidelberg, 2012, pp. 101-117.

${ }^{5}$ Withers, P.C., "An Aerodynamic Analysis of Bird Wings as Fixed Aerofoils," Journal of Experimental Biology, Vol. 90, No. 1, 1981, pp. 143-162.

${ }^{6}$ Alford Jr., W.J., Henderson, W.P., and Luoma, A.A., "Wind-Tunnel Studies at Subsonic and Transonic Speeds of a MultipleMission Variable-Wing-Sweep Airplane Configuration," NASA-TM-X-206, 1959.

${ }^{7}$ Hall, J., Mohseni, K., Lawrence, D. and Geuzaine, P., "Investigation of variable wing-sweep for applications in micro air vehicles," Infotech@Aeospace, AIAA, Arlington, VA, 2005.

${ }^{8}$ Pankonien, A. M., Duraisamy, K., Faria, C. T., \& Inman, D.., "Synergistic Smart Morphing Aileron: Aero-structural Performance Analysis," 22nd AIAA/ASME/AHS Adaptive Structures Conference, AIAA SciTech Forum, National Harbor, Maryland, 2014.

$>{ }^{9}$ Bilgen, O., Kochersberger, K. B., Inman, D. J., \& Ohanian, O. J., "Novel, Bidirectional, Variable-camber Airfoil via MacroFiber Composite Actuators," Journal of Aircraft, Vol. 47, No. 1, 2010, pp. 303-314.

$\checkmark 10$ Pankonien, A.M., Gamble, L.L., Faria, C.T., and Inman, D.J., "Synergistic Smart Morphing Aileron: Capabilities Identification,” 24th AIAA/AHS Adaptive Structures Conference, AIAA SciTech Forum, San Diego, California,, 2016.

American Institute of Aeronautics and Astronautics 